\title{
Comparative Analysis of the Relationship between Job Burnout and General Health of Male and Female High School Teachers in Zabol
}

\author{
Tahere Abkhou', Hossein Jenaabadi ${ }^{*}$ \\ ${ }^{1}$ MA Student of Educational Psychology, Department of Psychology, Zahedan Branch, Islamic Azad University, \\ Zahedan, Iran \\ ${ }^{2}$ Faculty of Educational Sciences and Psychology, University of Sistan and Baluchestan, Zahedan, Iran \\ Email: $\underline{\text { hjenaabadi@ped.usb.ac.ir }}$
}

Received 2 November 2014; accepted 29 April 2015; published 5 August 2015

Copyright (C) 2015 by authors and Scientific Research Publishing Inc.

This work is licensed under the Creative Commons Attribution International License (CC BY).

http://creativecommons.org/licenses/by/4.0/

(c) (i) Dpen Access

\begin{abstract}
The aim of this study was to determine the relationship between general health and job burnout of male and female high school teachers in Zabol. The research followed a cross-correlational method. The statistical population included all male and female high school teachers in the academic year 2014 in Zabol. In this study, considering participants' gender, stratified random sampling method was used. Data collecting was performed through applying the Maslach's Burnout Inventory and General Health Questionnaire (GHQ-28). The content validity was checked and alpha coefficients for the variables of general health and job burnout were 0.92 and 0.81 , respectively. In this research study, Pearson correlation coefficient, regression analysis, and independent t-test were conducted to analyze the hypotheses using SPSS v.21. Results revealed that general health and its dimensions were significantly and positively correlated with job burnout of male and female high school teachers in Zabol. Additionally, emotional exhaustion and depersonalization were significantly and negatively related to general health. Regarding the priority of the predictor variables of teachers' general health, in the first step, emotional exhaustion, in the second step, depersonalization and in the third step, lack of personal accomplishment entered into the prediction model, these three variables together predicted $21 \%$ of variations in general health. Moreover, general health and job burnout were not significantly correlated in terms of gender.
\end{abstract}

\section{Keywords}

General Health, Job Burnout, Teachers

\footnotetext{
${ }^{*}$ Corresponding author.
}

How to cite this paper: Abkhou, T. and Jenaabadi, H. (2015) Comparative Analysis of the Relationship between Job Burnout and General Health of Male and Female High School Teachers in Zabol. Natural Science, 7, 391-399. 


\section{Introduction}

In recent years, there has been a growing interest in studying the phenomenon of job burnout among researchers and those involved in labor productivity. This is mainly due to the perceived negative effects of job burnout on work forces. Job burnout is a damage to mental health, which is formed in connection with the job and has three dimensions: 1) emotional exhaustion, which is completely similar to stress and the feeling of being under pressure and leads to the elimination of the person's emotional resources; 2) depersonalization, which is an inexorable and negative response to those who are usually the receiver of the person's service. This dimension refers to negative perceptions of others; and 3) reduction of personal performance, which is followed by a decrease in the feeling of competency in performing personal responsibility and a negative self-evaluation regarding tasks [1].

Nowadays, people who are in different conditions and various positions feel some degree of job burnout in their working environment and this sense of reality is something tangible and undeniable in modern organizations [2]. Job burnout is one of the results of various pressures occurring in the form of psychological and physical symptoms [3].

WHO defines job burnout as a psychological process that occurs under conditions of extreme stress and is reflected as emotional exhaustion, depersonalization, loss of motivation and deterioration (weak performance) [4]. Job burnout is a state of chronic emotional state including three components of cognitive, physical and emotional fatigue [5]. Job burnout could be defined as a consequence of chronic reduction of one's ability resources arising from long-term exposure to stress, especially job stressors. Although initial research focused on negative organizational, attitudinal and psychological consequences [6] [7], recent evidence shows that job burnout can negatively affect physical health and in addition it is known as a risk factor for physical illness and somatic disorders [8].

Teachers, because of the nature of their job, are exposed to many problems, such as voluminous work, academic decline of students, inadequate wages and benefits, lack of students' interest in studying, ignoring their needs by educational organizations and overcrowded classrooms. These problems have a great impact on their health and continuance of these issues raises their vulnerabilities and ultimately may lead to job burnout [9].

Nasti Zaei, Vaezi and Molazehi investigated the relationship between job burnout and general health among the employees of Zahedan Department of Telecommunications. Results revealed that $15.4 \%$ and $23.4 \%$ of employees were respectively at risk of considering their job burnout and general health. Also, significant correlations were observed between job burnout and general health and their dimensions, between emotional exhaustion and anxiety and insomnia and general health, between depersonalization and general health, between anxiety and insomnia and job burnout, and between lack of personal accomplishment and impaired social relationships. Based on the clear relationship of job burnout and general health, the researchers suggested that managers needed to conduct programs to improve motivation, job satisfaction, sense of self-efficacy, and human relations [10].

Saberi, et al. explored the relationship between job burnout and general health of judges and prosecutors working in courts of Tehran. Result indicated a significant negative relationship between job burnout and general health, i.e. increased burnout was followed by decreased mental health [11].

Rostami, et al., examining the relationship of job burnout and mental health with controlling roles of gender and resiliency among primary school teachers, also stated that there was a significant relationship between job burnout and general health of individuals, in such a way that an increase in burnout reduced general health [12].

Shakerinia et al. examined the relationship of job stress and resilience with job burnout among female nurses and concluded that there was a significant positive correlation between job stress and burnout among nurses [13].

Jamali, et al. discussed organizational climate, job burnout, job satisfaction and its relationship with organizational citizenship behavior and found out that after variables of job satisfaction and job burnout, organizational climate had the major effect on organizational citizenship behavior [14].

Ahghar explored the role of organizational culture on job burnout of teachers in middle schools in Tehran. Result of this research indicated that organizational culture scores could predict teaches' job burnout and also the predictive power of entrepreneurial culture was more than that of participatory culture, while the predictive role of participatory culture was more than that of both bureaucratic and missionary cultures [15].

Boshlideh, Taghipour, Hashemi Sheikhshabani, and Jazaieri examined the relationship between job burnout and general health of middle school teachers. Results showed that the total score of job burnout and its dimen- 
sions were significantly correlated with the total score of general health and its dimensions. Additionally, the dimension of emotional exhaustion affected teachers' general health more than other dimensions of job burnout. Given the high correlation between emotional exhaustion and general health, the researchers suggested that teachers should be protected against damages of burnout, especially the dimension of emotional exhaustion [16].

Ghobaribanab, Khazaei, Alizadeh, and Tofi found a significant difference between teachers in normal school and teachers in exceptional schools. No significant difference was observed considering their gender and educational level and job burnout. Meanwhile the young teachers experienced more job burnout than their old counterparts. They also examined causes of conflicts in teachers and managers' behavior and its relationship with academic improvement, motivation improvement and psychosomatic characteristics of students and found three factors of job burnout, job satisfaction and control ideology among the main causes [17].

Ghadimi Moghaddam and Hosseini Tabatabaee compared the prevalence of job burnout syndrome among teachers and administrative staff of the Education Department in the province of Kerman ( $\mathrm{N}=1068)$ and concluded that male teachers and staff, compared to female teachers, experienced more job burnout and high school teachers suffered more from job burnout compared to elementary school teachers [18].

Gargari has shown the levels of teachers' job burnout: in male teachers, high (5.7\%) and moderate emotional exhaustion (25.14\%), high (32.11\%) and moderate depersonalization (2.13) and high (16.47\%) and moderate lack of personal accomplishment (18.30\%) and in female teachers, high (4.3\%) and moderate emotional exhaustion (95.7\%), high (4.03\%) and moderate depersonalization (5.12) and high (95.32\%) and moderate lack of personal accomplishment (19.3\%). Additionally, findings about the impact of coping styles on job burnout indicated a significant difference between male and female teachers' emotional exhaustion and problem-centered coping mechanisms (problem solving, positive reappraisal, and support seeking), and also between male and female teachers' emotional exhaustion and emotion-centered coping mechanisms (avoidance, escape and encounter). The author also found a significant difference between depersonalization of male and female teachers who adopted problem-centered as well as emotion-centered coping mechanisms, and a significant difference considering lack of personal accomplishment for male teachers who adopted problem-centered as well as emotion-centered coping mechanisms. However, there was no significant difference in the case of female teachers [19].

Maunz indicated that mental health problems were significantly higher in individuals with job burnout [20].

Sidman and Zager found that many of teachers' mental and physical problems were associated with job burnout [21].

Nayoung, Eunkyoung, Hyunjung, Eunjoo, \& Sang Min’s meta-analysis on occupational and individual factors influencing job burnout showed that job-related factors such as age were the most significant indicators of depersonalization, emotional exhaustion. In relation to the level of personal accomplishment, variables of age and working hours were the most significant indicators with positive correlation [22]. Therefore, the researchers suggested the necessity of attention to the effect of job-related factors on mental health issues and prevention of job burnout.

\section{Research Methods}

In this study, the descriptive method (cross-correlational) was applied.

The statistical population of this study included all male and female high school teachers in the academic year 2014 in Zabol.

As Table 1 shows, considering participants' gender, stratified random sampling method was conducted, i.e. among 749 teachers, 152 were selected to complete the questionnaires.

The sample size of this research was determined on the basis of the Morgan's table. Therefore, data analysis conducted with 152 subjects (102 female teacher and 50 male teachers).

Table 2 shows that 33\% of the sample included a total of 50 male teachers and 67\% included 102 female teachers. The total number of subjects is 152 .

\section{Materials}

Data collection was conducted through applying the following questionnaires.

a) Maslach's Burnout Inventory (MBI): The test was developed by Maslach [23]. It is a new estimate of the phenomenon of stress, i.e., exhaustion. The reliability of MBI subscales, using Cronbach's alpha, was reported 
Table 1. Frequency of male and female high school teachers in Zabol.

\begin{tabular}{cccc}
\hline Gender & Male & Female & Total \\
\hline $\mathbf{N}$ & 87 & 173 & 256 \\
\hline
\end{tabular}

Table 2. Frequency of the sample for male and female teachers.

\begin{tabular}{ccc}
\hline Gender & N & Percent \\
\hline Male & 50 & 33 \\
Female & 102 & 67 \\
Total & 152 & 100 \\
\hline
\end{tabular}

as 0.71 to 0.90 . The reliability of the test in research studies with Iranian samples is satisfactory [24]. The inventory consists of 22 items that measure emotional exhaustion, depersonalization and lack of personal accomplishment within the framework of professional activities. It is especially used to measure and prevent job burnout among nurses and teachers, etc. The scoring procedure is performed based on a 7-point Likert scale including never, a few times a year or less, once a month or less, a few times a month, once a week, a few times a week, and every day.

b) General Health Questionnaire (GHQ-28): GHQ-28 was developed by Goldberg and Hiller [25]. It includes 4 subscales, each consisting of 7 items. The scales are as follows:

1) Somatic symptoms;

2) Anxiety and insomnia;

3) Social dysfunction;

4) Depression.

Items 1 to 7,8 to 14,15 to 21, and 22 to 28 measure somatic symptoms, anxiety and insomnia, social dysfunction and depression, respectively. To sum the scores $a$ receives zero, $b$ receives 1 , and the like. In each of the subscales, a score of 6 and higher and in the total scale, a score of 22 and higher reflects general health symptoms.

To calculate the reliability of these questionnaires, copies of them were distributed among 30 subjects. Cronbach's alpha was used to calculate the reliability of the test. The following table shows the coefficient alpha for each of the questionnaires.

Table 3 shows that the alpha coefficient of general health and job burnout is $92 \%$ and $81 \%$, respectively.

In this research study, Pearson correlation coefficient, regression analysis, and independent t-test were conducted to analyze the hypotheses using SPSS v.21.

\section{Results}

H1: There is a relationship between general health and its dimensions and job burnout among male and female high school teachers in Zabol.

Table 4 shows a negative and significant relationship between job burnout and general health $(\mathrm{r}=-0.28, \mathrm{p}<$ 0.01 ) at the $99 \%$ confidence level. Thus, those who have high job burnout, have less general health and vice versa. Also, the relationship between job burnout and somatic symptoms, anxiety and depression is positive and significant; i.e. higher job burnout leads to increase in these symptoms.

H2: There is a relationship between burnout and its dimensions and general health among male and female high school teachers in Zabol.

Table 5 shows a negative and significant relationship between job burnout and general health $(\mathrm{r}=-0.28$, $\mathrm{p}<$ 0.01 ) on the $99 \%$ confidence level. Thus, those who have high depersonalization and emotional exhaustion have less general health. However, the relationship between general health and lack of personal accomplishment is not significant $(\mathrm{r}=-0.12)$.

H3: Job burnout dimensions can predict general health among male and female high school teachers in Zabol.

As Table 6 shows, in the first step, emotional exhaustion was a better predictor of general health. This variable alone could predict $17 \%$ of the variations in general health. In the second step, depersonalization entered into the prediction model and these two variables were able to predict $20 \%$ of the variations in general health. In the 
Table 3. Alpha coefficients to determine the reliability of variables.

\begin{tabular}{ccc}
\hline Variable & The number of Items & Alpha \\
\hline General health & 28 & 0.92 \\
Burnout & 22 & 0.81 \\
\hline
\end{tabular}

Table 4. Correlation coefficient of general health and its dimensions with job burnout.

\begin{tabular}{ccc}
\hline Index variable & r & Sig \\
\hline Burnout-somatic symptoms & 0.27 & 0.000 \\
Burnout-anxiety & 0.22 & 0.000 \\
Burnout-social dysfunction & 0.17 & 0.00 \\
Burnout-depression & 0.23 & 0.000 \\
Burnout-general health & -0.28 & 0.000 \\
\hline
\end{tabular}

Table 5. Correlation coefficient of job burnout and its dimensions with general health.

\begin{tabular}{ccc}
\hline Index variable & R & Sig \\
\hline General health-emotional exhaustion & -0.24 & 0.000 \\
General health-lack of personal accomplishment & -0.12 & 0.12 \\
General health-depersonalization & -0.20 & 0.00 \\
General health-burnout & -0.28 & 0.000 \\
\hline
\end{tabular}

Table 6. Correlation coefficient of job burnout and its dimensions with general health.

\begin{tabular}{|c|c|c|c|c|c|c|}
\hline Step & Variable & $\mathbf{R}^{2}$ & $\mathbf{F}$ & $\boldsymbol{\beta}$ & $\mathbf{t}$ & Sig \\
\hline 1 & Emotional exhaustion & 0.17 & 98.07 & 0.40 & 10.46 & 0.000 \\
\hline \multirow[t]{2}{*}{2} & $\begin{array}{l}\text { Emotional exhaustion } \\
\text { + depersonalization }\end{array}$ & 0.20 & $\begin{array}{l}98.07 \\
17.74\end{array}$ & $\begin{array}{l}0.40 \\
0.20\end{array}$ & $\begin{array}{c}10.46 \\
6.12\end{array}$ & 0.000 \\
\hline & $\begin{array}{c}\text { Emotional exhaustion } \\
+ \text { depersonalization }+ \\
\text { lack of personal } \\
\text { accomplishment }\end{array}$ & 0.21 & $\begin{array}{c}98.07 \\
17.74 \\
5.25\end{array}$ & $\begin{array}{l}0.40 \\
0.20 \\
0.19\end{array}$ & $\begin{array}{c}10.46 \\
6.12 \\
2.29\end{array}$ & 0.02 \\
\hline $\mathbf{N}=$ & $2, \mathrm{p}<0.01$ & & & & & \\
\hline
\end{tabular}

third step, lack of personal accomplishment entered into the prediction model and these three variables were able to predict $21 \%$ of the variations in general health. This means that this variable alone could predict $1 \%$ of the variations.

H4: There is a significant difference between general health and job burnout among teachers considering their gender.

Table 7 shows that the calculated t (0.53) is not significant at the 95\% confidence level $(\mathrm{df}=150)$; therefore, it can be concluded that the level of general health of male and female teachers is the same. In addition, no significant difference was observed between male and female teachers' job burnout $(\mathrm{p}<0.05, \mathrm{t}=-0.64)$.

\section{Discussion}

H1: There is a relationship between general health and its dimensions and job burnout among male and female high schools teachers in Zabol.

The main objective of the study was to investigate the relationship between burnout and general health of 
Table 7. Difference in teachers’ general health and job burnout considering their gender.

\begin{tabular}{cccccccc}
\hline Variables & Gender & N & Mean & SD & t & df & Sig \\
\hline General health & Male & 50 & 19.12 & 12.7 & & & \\
& Female & 102 & 17.40 & 6.25 & 0.53 & 150 & 0.43 \\
Burnout & Male & 50 & 41.80 & 12.8 & & & 150 \\
& Female & 102 & 44.35 & 13.88 & -0.64 & 0.32 \\
\hline
\end{tabular}

teachers. Results of Pearson correlation indicated a significant negative relationship between job burnout and general health. A significant relationship existed between job burnout and general health dimensions; therefore, an increase in job burnout decreased general health. In line with these results, Rostami also stated that there was a significant relationship between individuals’ job burnout and general health, so that increasing job burnout reduces general health. Moreover, Bahri Binabaj et al. [26], Nasti Zaei, Vaezi and Molazehi [10], Abdi Masuleh et al. [27], Boshlideh, et al. [16], Sidman and Zager [21] in their studies have concluded that general health was lower in people who have higher job burnout. Since job burnout causes emotional, physical and psychological symptoms, it may affect individuals’ health.

H2: There is a relationship between burnout and its dimensions and general health among male and female high schools teachers in Zabol.

The results of Pearson correlation indicated a significant negative relationship between emotional exhaustion and depersonalization and general health. Therefore, it can be argued that those who have high emotional exhaustion, are low in general health. This finding is consistent with Nasti Zaei, Vaezi and Molazehi [10], Arizi, Nouri, Zare, and Amiri [1], Abdi Masuleh et al. [27], Jamali, et al. [14], Ahghar [15], Boshlideh, et al. [16], Maritn [28], and Lackritz [29]. Shirom, Melamed, Toker, Berliner, and Shapira, Melamed [8], who found a significant negative relationship between emotional exhaustion and general health. Therefore, given the theoretical foundations and results obtained from research studies, it could be concluded that emotional exhaustion, as a threatening factor, affects teachers' health general.

Additionally, the results of Pearson correlation indicated a significant negative relationship between depersonalization and general health and its subscales. The finding is not consistent with the results of Ghobadi [30] who concluded that there was not any relationship between depersonalization and general health, and also with the results of Mostahfezian et al. [31] who indicated that there was not any significant relationship between social dysfunction and depersonalization. However, the result is in line with those of Mohammadi [32], Saberi [11], Boshlideh, et al. [16], Martin [28] who found a significant relationship between depersonalization and general health. Accordingly, it could be argued that those who have high depersonalization are low in general health.

In addition, the results of Pearson correlation indicated that there was not any significant relationship between lack of personal accomplishment and general health. This is not consistent with the results of research conducted by Ghobadi [30], Abdi Masouleh et al. [27] and Nasti Zaei [10] who indicated that lack of personal accomplishment was significantly correlated with general health subscales and if it increases, general health decreases.

H3: Burnout dimensions can predict general health among male and female high school teachers in Zabol.

According to the regression results, in the first step, emotional exhaustion was a better predictor of general health. This variable alone could predict $17 \%$ of the variations in general health. In the second step, depersonalization entered into the prediction model and these two variables were able to predict $20 \%$ of the variations in general health. This means that this variable alone could predict 3\% of the variations. In the third step, lack of personal accomplishment entered into the prediction model and these three variables were able to predict $21 \%$ of the variations in general health. This means that this variable alone could predict $1 \%$ of the variations.

H4: There is a significant difference between general health and burnout among teachers with regard to their gender.

Results indicated that calculated t (0.53) was not significant at the 95\% level ( $\mathrm{df}=150)$; therefore, it could be concluded that the level of general health of male and female teachers was the same. The finding was in line with Beer [33] who indicated higher levels of psychiatric disorders among women than men, and with the results of Moradi et al. [34], Sadeghi Boroujerdi [35] and Habibi et al. [36] who found that women have lower mental and physical health and also their psychological distress is more than that of male teachers. However, the finding is not consistent with the findings of Gust and Dewe [37], and Rostami et al. [12] who have shown that male 
teachers' scores on anxiety, depression and somatic symptoms of the GHQ were higher than those of female teachers.

In addition, no significant difference was observed between male and female teachers' job burnout ( $\mathrm{p}<0.05, \mathrm{t}$ $=-0.64)$. In other words, male and female teachers were similar in terms of the level of job burnout. The finding is consistent with the results of Navidian [38] who concluded that there was no significant difference between male and female subjects in terms of job burnout and both genders experienced the same level of occupational stressors. However, it is not consistent with the results of Jafarpoor [39] and Keshani [40] who found that men suffered more from job burnout compared to women, and this difference was statistically significant. The result is also inconsistent with the results Beer [33], Rostami, et al. [12] who found a significant difference between male and female subjects in terms of job burnout and exhaustion and concluded that women suffered more from job burnout compared to men.

\section{Conclusions}

Enhancing the efficiency and productivity of staff like other resources requires their enjoyment from high mental and physical health, happiness and peace which depend on a healthy and vibrant life and optimal use of time and leisure to ensure general health. Therefore, organizations that are interested in improving the quality of life for their labor force try to create a sense of freshness, dynamism and vitality in employees [41].

Strong relationships of job burnout with general health encompass useful applicable themes for protecting work force and optimal protection of work environments. It is recommended that teachers' job burnout status should be annually evaluated and effective interventions should be provided to control the underlying factors. Equipping organizations with medical staff and tracing symptoms of job burnout can help teachers' psychological well-being and in general staff of an organization. As a result, such support will be effective in providing mental health for staff. Since teachers are messenger of education, their health is important; because teachers who suffer from low general health cannot nurture good students for the future of the society.

To sum up, it could be argued that there was a negative relationship between job burnout and general health of high school teachers in Zabol. In other words, teachers with high job burnout have lower general health. The level of general health of male and female teachers was similar and also no significant difference was observed between male and female teachers in terms of job burnout and its subscales (emotional exhaustion, depersonalization and lack of personal accomplishment), and emotional exhaustion had the most predictive power for general health.

\section{References}

[1] Oreyzi, H., Nouri, A., Zare, R. and Amiri, M. (2013) Meta-Analysis of the Researches Done about the Relationship between Burnout and Mental Health. Social Welfare, 13, 93-112.

[2] Weightman, J. (1999) Introduction Organization Behavior. Longman Press, London.

[3] Russell, D.W., Altmaier, E. and Van Velzen, D. (1987) Job-Related Stress, Social Support, and Burnout among Classroom Teachers. Journal of Applied Psychology, 722, 269-274. http://dx.doi.org/10.1037/0021-9010.72.2.269

[4] World Health Organization (1998) The World Health Report 1998_-Life in the 21st Century: A Vision for All.

[5] Shirom, A. (2003) Job-Related Burnout: A Review. In: Quick, J.C. and Tetrick, L.E., Eds., Handbook of Occupational Health Psychology, American Psychological Association, Washington DC, 245-264. http://dx.doi.org/10.1037/10474-012

[6] Cordes, C.L. and Dougherty, T.W. (1993) A Review and an Integration of Research on Job Burnout. Academy of Management Review, 18, 621-656.

[7] Maslach, C., Schaufeli, W. and Liter, M.P. (2001) Job Burnout. Annual Review of Psychology, 52, 397-422. http://dx.doi.org/10.1146/annurev.psych.52.1.397

[8] Shirom, A., Melamed, S., Toker, S., Berliner, S. and Shapira, I. (2005) Burnout and Health: Current Knowledge and Future Research Directions. In: Anthoniou, A. and Cooper, C., Eds., New Perspectives in Occupational Health, Vol. 20, 269-309. http://dx.doi.org/10.1002/0470029307.ch7

[9] Honkonen, T., Ahola, K., Pertovaara, M., Isometsä, E., Kalimo, R., Nykyri, E., Aromaa, A. and Lönnqvist, J. (2004) The Association between Burnout and Physical Illness in the General Population-Results from the Finnish Health 2000 Study. Journal of Psychosomatic Research, 61, 59-66. http://dx.doi.org/10.1016/j.jpsychores.2005.10.002

[10] Nasti Zaei, N., Vaezi, M.D. and Molazehi, A. (2008) The Relationship between Job Burnout and General Health of 
Employees of Department of Telecommunications in Zahedan. Yazd Health Research Quarterly, 7.

[11] Saberi, S.M., et al. (2008) The Relationship between Burnout and General Health of Judges and Prosecutors Working in the Judicial Authorities in Tehran. Journal of Legal Medicine, 14, 92-98.

[12] Rostami, A.R., et al. (2008) Exploring the Relationship of Job Burnout and Psychological Wellbeing with the Controlling Role of Gender and Resiliency among Primary Teachers. Quarterly of Iran Occupational Health, 5, 68-75.

[13] Shakerinia, I. and Mohammadpour, M. (2010) Relationship between Job Stress and Resiliency with Occupational Burnout among Nurses. Journal of Kermanshah University of Medical Sciences, 14, 161-169.

[14] Jamali, D., Zanhour, M. and Keshishian, T. (2009) Peculiar Strengths and Relational Attributes of SMEs in the Context of CSR. Journal of Business Ethics, 87, 355-367. http://dx.doi.org/10.1007/s10551-008-9925-7

[15] Ahghar, G.H. (2006) The Role of School Organizational Culture in Teachers' Job Related Fatigue. Journal of Education, 86, 93-123.

[16] Boshlideh, K., Taghipour, M., Hashemi Sheikhshabani, I. and Jazaieri, Z. (2011) The Relationship between Job Burnout and General Health of Middle School teachers. Journal of Practical Advice of Shahid Chamran University, 1.

[17] Ghobaribanab, B., Khazaei, M.A., Alizadeh, H. and Tofi, Z. (2004) Determine the Predicators of Job Burnout (Teachers of Normal and Retarded Children in Birjand). News Journal of Psychotherapy, 9, 33-34.

[18] Ghadimi Moghaddam, M.M. and Hosseini Tabatabaee, F. (2006) Prevalence of Burnout Syndrome and Its Relationship with Gender, Education Level, Job Classification, and Geographical Location among Teachers and Employees of the Education Organization. Psychological Research, 9, 56-73.

[19] Badri Gargouri, R. (1995) Psychological Syndrome and Job Burnout of Teachers and Coping Mechanisms. Master’s Thesis, Tarbiat Modares University, Tehran.

[20] Ehyakonandeh, M., Shafiabadi, A.A. and Sudani, M. (2008) The Relationship between Health and Job Burnout of the Staff of Islamic Azad University. Journal of Thought and Behavior, 3.

[21] Gargari, B. (1995) Psychological Syndrome of Teachers’ Job Burnout and Coping Mechanisms. Master Thesis of Psychology, Tarbiat Moddares University, Tehran.

[22] Lim, N., Kim, E.K., Kim, H., Yang, E. and Lee, S.M. (2010) Individual and Work-Related Factors Influencing Burnout of Mental Health Professionals: A Meta-Analysis. Journal of Employment Counseling, 47, 86-96.

[23] Maslach, C. and Jackcon, S.E. (1981) The Maslach Burnout Inventor Research Edition. Counseling. Psychologist Press, Palo Alto.

[24] Shokrkon, H. (1995) Sources of Resistant against Mental Pressure. Proceedings of the First Seminar on Stress, Allameh Tabatabei University, Tehran.

[25] Goldberg, D.P. and Hillier, V.F. (1979) A Scaled Version of the General Health Questionnaire. Psychological Medicine, 9, 139-145. http://dx.doi.org/10.1017/S0033291700021644

[26] Bahri Binabaj, N., Moghiman, M., Atarbashi, M. and Garche, M. (2003) The Survey of Relationship between Job Burnout and Health Status of Midwives and Nurses. Ofogh-e-Danesh, 9, 99-104. (In Persian)

[27] Abdi Masouleh, F., Kaviani, H., Haghanizadeh, M. and Momeni Araghi, A. (2007) The Relationship between Burnout and Mental Health among Nurses. Tehran University Medical Journal, 65, 65-75.

[28] Martin, S.S. (2008) Relational and Economic Antecedents of Organizational Commitment. Personnel Review, 37, 589608. http://dx.doi.org/10.1108/00483480810906856

[29] Lackritz, J.R. (2004) Exploring Burnout among University Faculty: Incidence, Performance, and Demographic Issues. Teaching and Teacher Education, 20, 713-729. http://dx.doi.org/10.1016/j.tate.2004.07.002

[30] Ghobadi, A. (2006) Examining the Relationship between General Health and Job Burnout among Physical Education Teachers in Kermanshah. Master’s Thesis, Razi University of Kermanshah, Kermanshah.

[31] Mostahfezian, M., Mozafari, A.A. and Amirtash, A.M. (2008) Comparison Study of General Health, Quality of Work Life of Active and Inactive Faculty Members at Different Azad Universities in Area 4. Research on Sport Sciences, 4, 105-108.

[32] Mohammadi, S.H. (2006) Teachers’ Burnout and Psychological Health. Journal of Iranian Psychologists, 3, 15-23.

[33] Beer, J. (1992) Burnout and Stress, Depression and Self-Esteem of Teachers. Psychological Research Experience Program, 71, 1331-1336.

[34] Moradi, A.H., Poursarrajian, D. and Alizadeh Naeeni, A.R. (2013) The Relationship between Hardiness and Burnout among the Teachers of the Universities and Higher Educational Institutes-Case Study. European Online Journal of Natural and Social Sciences, 2, 500-506.

[35] Sadeghi Boroujerdi, S. (2003) The Relationship of Job Stressors with Physical and Mental Health and Job Burnout of Faculty Members of Sports Science. Ph.D. Thesis, Faculty of Physical Education and Sports Science. 
[36] Habibi, E., Dehghan, H., Safari, S., Mahaki, B. and Hassanzadeh, A. (2014) Effects of Work-Related Stress on Work Ability Index among Refinery Workers. Journal of Education of Health Promotion, 3, 18. http://dx.doi.org/10.4103/2277-9531.127598

[37] Guest, D. and Dewe, P. (1990) Methods of Coping with Stress at Work: A Conceptual Analysis and Empirical Study of Measurement Issues. Journal of Organizational Behavior, 11, 135-150. http://dx.doi.org/10.1002/job.4030110205

[38] Navidian, A. (2005) Examining Occupational Stressors and Their Relationship with General Health of Nurses Working in Hospitals in Zahedan. Scientific and Research Journal of Kermanshah University of Medical Sciences, 9.

[39] Jafarpoor, H. (1997) The Relationship between Hardiness and Burnout in Teachers of Ordinary and Special Schools. Master's Thesis, Tehran University, Tehran.

[40] Keshani, A. (1998) Job Satisfaction and Job Burnout among the Teachers of Mentally Retarded Children. Master's Thesis of Psychology, Tehran University, Tehran.

[41] Saatchi, M. (2008) Mental Health in the Workplace with Emphasis on Job Stress and Burnout. Viraiesh, Tehran. 